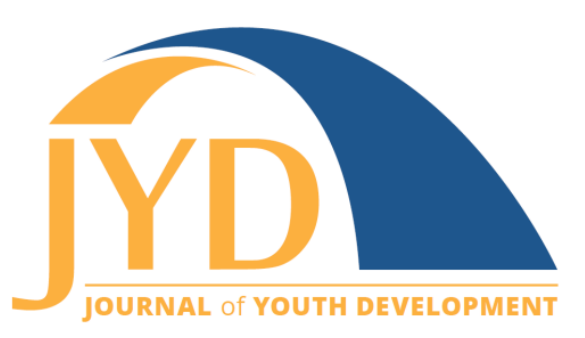

http://jyd.pitt.edu/ | Vol. 15 Issue 1 DOI 10.5195/jyd.2020.815 | ISSN 2325-4017 (online)

\title{
Betting on the Workforce: An Interview With the S. D. Bechtel, Jr. Foundation
}

\author{
Deborah A. Moroney \\ American Institutes for Research \\ dmoroney@air.org \\ Jill Young \\ American Institutes for Research \\ jiyoung@air.org
}

\begin{abstract}
In this article, Deborah Moroney and Jill Young from the American Institutes for Research interview Rebecca Goldberg and Alex Hooker, senior program officers from the S. D. Bechtel, Jr. Foundation. Rebecca and Alex describe their experience as members of the youth development workforce and how that experience inspired them to support the youth development workforce on the grantmaking side, focusing on adult practice and youth character development. As the S. D. Bechtel, Jr. Foundation sunsets in 2020, Rebecca and Alex also share their hopes for the youth development workforce going forward.
\end{abstract}

Key words: youth development, workforce, capacity building, adult practice, grantmaking

\section{Introduction}

Youth development is rooted in the idea that all young people should have the opportunity to thrive (Caldwell \& Witt, 2018). Thriving is what "happens when young people work with purpose to achieve their full potential" by engaging in their own unique talents, interests, and/or aspirations in purposeful ways (Caldwell \& Witt, 2018, p. 2). Adults play a critical role in providing opportunities for youth to engage in social and emotional learning (SEL) and to thrive in out-of-school time (OST) programs (Bocarro and Witt, 2018; National Academies of Sciences, Engineering, and Medicine, 2019). We know that factors such as staff training, intentional program practices, and adult competencies foster positive relationships and promote SEL and

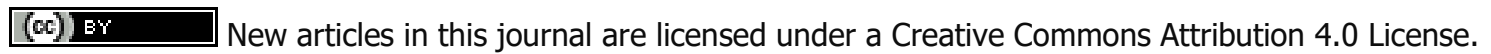
This journal is published by the University Library System, University of Pittsburgh and is cosponsored by the University of Pittsburgh Press. The Journal of Youth Development is the official peer-reviewed publication of the National Association of Extension 4-H Youth Development Professionals and the National AfterSchool Association. 


\section{Betting on the Workforce}

thriving in a variety of outcome areas (Durlak, Weissberg, Dymnicki, Taylor, \& Schellinger, 2011; Vandell, Larson, Mahoney, \& Watts, 2015).

The S. D. Bechtel, Jr. Foundation (the Foundation) launched a national initiative focused on building capacity of the youth development (YD) workforce. The American Institutes for Research (AIR) serves as one of the Foundation's partners. As the Foundation prepares to sunset in 2020, we interviewed Foundation senior program officers Rebecca Goldberg and Alex Hooker to learn more about their portfolios, which are aimed at building the capacity of the YD workforce and ultimately ensuring programs foster participants' character, social, and emotional development. This interview highlights the importance of the YD workforce in supporting youth learning and development, shares lessons learned and successful strategies for supporting the YD workforce, and offers hopes for the YD field.

\section{Background}

In this section, we briefly elevate relevant literature on the YD workforce to ground our discussion with Rebecca and Alex.

Lessons learned from collaborative efforts, such as the Aspen Institute National Commission on Social, Emotional, and Academic Development (SEAD); the Science of Learning and Development (SoLD) Alliance; and the National Academies of Science, Engineering, and Medicine underscore the importance of supporting all young people to thrive, and on the road to thriving (among other factors) is building character and supporting social and emotional learning (SEL). Intentional opportunities for SEL support learning and development and facilitate skill building, academic achievement, and success in life (Durlak et al., 2011). Character development is another skill-building approach aligned with SEL, with some marked differences. Character development is the process through which youth develop "a sense of fairness, responsibility...among other attitudes and skills" (Moroney \& Devaney, 2017, p. 68).

OST programs can play a role in supporting and facilitating young people's learning and development, including SEL and character development (National Academies of Sciences, Engineering, and Medicine, 2019). OST programs provide settings for youth to "experience supportive social relationships with both adults and peers, relationships that foster emotional, behavioral, and cognitive development" (National Academies of Sciences, Engineering, and Medicine, 2019, p. 69). The contexts and relationships in which young people learn and develop 


\section{Betting on the Workforce}

affects their growth (Aspen Institute National Commission on Social, Emotional, and Academic Development, 2019; National Academies of Sciences, Engineering, and Medicine, 2019; Science of Learning and Development Alliance, 2019); young people need "high-quality learning environments where each [youth] experiences safety, belonging, and purpose" in order to develop (Aspen Institute National Commission on Social, Emotional, and Academic Development, 2019, p. 40). In after-school programs in OST settings, high-quality programs are

- culturally responsive, strengths-based, and built from individual assets

- fortified by strong management practices

- led by qualified and trained staff

- supported by strong partnerships with schools, families, and the community

- warm, welcoming, and supportive of relationship building

- structured to provide opportunities for intentional skill building

- youth-driven, with authentic opportunities for youth voice, choice, leadership, and agency

- evaluative, striving for continuous improvement (Moroney, Newman, \& Osher, 2018).

As the Foundation observed, the YD workforce is key to implementing high-quality programs that foster SEL and character development, and youth development more broadly (Aspen Institute National Commission on Social, Emotional, and Academic Development, 2019; DarlingHammond, Flook, Cook-Harvey, Barron \& Osher, 2019; Moroney \& Devaney, 2017). The YD workforce "influences the quality of the experiences young people have in programs, and they play an important role in the lives of young people by being a caring adult offering a wide variety of experiences that positively impact their lives" (Borden \& Perkins, 2006, p. 102). We recognize the importance of "warm, stable, responsive, and supportive relationships with adults and peers" drive learning and development in OST and other settings (American Institutes for Research, 2019, p. 3). Given the importance of the YD workforce, there has been increased attention on how we prepare and develop the adults who work with young people (Borden \& Perkins, 2006). The Foundation recognized an opportunity to support and build the capacity of the YD workforce and ultimately improve youth outcomes.

We, as longtime practitioners and researchers in $Y D$, applauded the Foundation's efforts to support and build the capacity of the YD workforce and were eager to hear what Rebecca and Alex learned from their experience. In many cases, Alex and Rebecca's good reflections are culled from or codify the literature. Where this is evident, we cite their thinking to show the 


\section{Betting on the Workforce}

alignment between the Foundation's initiative and the YD literature. Let's launch into the interview.

Rebecca and Alex, both of you have experience as members and supporters of the YD workforce. Tell us about your background and what inspired you to invest in the YD workforce.

Rebecca: I started my career in California seeking opportunities to learn about and support education and ultimately help kids thrive. As I was finishing up a postcollege internship at Sesame Workshop in New York City and was deciding between two very different job opportunities (one in conference planning and the other in a statewide organization supporting after-school professionals), my mentor at Sesame Workshop said, "After-school is where it's at! That's where kids get to be kids." Based on that advice, I started learning about YD at the California School-Age Consortium (CaISAC). I believe that after-school programs are impactful because they create the environments and provide role models for youth to be themselves, discover their passions, and figure out who they want to be in life.

In this position, I learned about the crucial role staff play in supporting young people's development. Since then, I've been a member and supporter of the YD workforce through my work at CalSAC, a state intermediary in California and a community-based organization in Los Angeles. I've supported the YD workforce in many ways: organizing conferences for afterschool professionals, supporting trainings, and experimenting with ways to support the YD workforce by building the pipeline of workers as well as developing career pathways into teaching.

Then, I found my dream position at the Foundation as a program officer in its national character initiative. At the Foundation, I co-lead a $\$ 130$ million strategy focused on improving adult practice in national YD organizations that are committed to character development and SEL. In addition to the national character initiative, I developed a California portfolio focused on supporting quality improvement in after-school programs while infusing SEL practices in partnership with the California Department of Education and several intermediaries.

Alex: I started my career working with youth through the National Park Service, where I learned about environmental education and other nature-based programming used to engage the local community through hands-on habitat restoration activities. My formal education and skillset were in conservation biology and I had no formal training in YD, but I understood the value in 


\section{Betting on the Workforce}

team building and other interpersonal activities to keep youth engaged in the program and develop a positive relationship with nature and the national parks.

I worked in the Golden Gate National Parks, which is different from most national parks in that it exists in a dense urban area and is used by many youth-serving organizations. In fact, there is a YMCA branch, a YMCA camp, and other nationally branded YD organizations within the park boundaries. I experienced many of these programs, learning new YD practices for my own nature-based programs. We eventually formalized peer-learning opportunities between staff in the youth-serving organizations in the park, which was the first time I identified as a youth worker-a big transition from my original identity as a conservationist. Since this experience, I have continued to support nontraditional youth practitioners like environmental educators to learn the best practices and craft wisdom coming from the YD workforce.

I joined the Foundation in 2012 to manage a portfolio of traditional and nontraditional environmental literacy organizations in the San Francisco Bay Area focused on using data to improve field staff practices. This work focused on improving participants' academic, YD, and environmental outcomes. The opportunity to manage the national character initiative with Rebecca allows me to use the YD skills I developed at the Golden Gate National Parks and research-backed YD practices that I learned through my environmental literacy work at the Foundation. Nature-based programming continues to be a core passion of mine and is represented in our national character initiative by calling out the power of nature in building young people's social and emotional skills and character.

Recently, the Foundation made a commitment to supporting the YD workforce. Can you tell us how the Foundation came to focus on this important area?

Both: The Foundation's Education strategy focuses on science, technology, engineering, and mathematics (STEM) and character development, which includes the national character initiative. Both the STEM and national character initiative were designed to focus on equipping the adult educator (i.e., the workforce), whether a teacher or a youth worker, with the skills and professional development needed to support young people in their education and development.

Because the Foundation was focused on large, complex problems related to education and the environment, our Board of Directors decided to spend down the Foundation's assets by 2020 to 


\section{Betting on the Workforce}

make a significant impact in these areas. The Board felt a sense of urgency to address issues such as the droughts in California; encouraging more young people to go into STEM fields; and the need to build SEL skills and character in young people who we are depending on to be the leaders that will solve the problems of tomorrow. Spending down the Foundation's assets shifted the grantmaking strategy from addressing local issues to making significant and strategic grants (our big bets) in California and nationally. This was particularly true for the Foundation's youth development work which moved the Foundation from funding in the San Francisco Bay Area to funding a national character initiative.

The Foundation realized that character development and SEL are inextricably linked (Moroney \& Devaney, 2017). Instinctively, and through research, the Foundation knew that both in and out of the classroom, adults serve as important mentors and role models for youth (Borden \& Perkins, 2006; Moroney \& Devaney, 2017). With only 5 years to launch and implement the Foundation's national character initiative, the Foundation decided to focus on improving adult practice of staff and volunteers, because we believed that by shifting adult behavior, measurable impacts on youth would follow. However, we knew we did not have enough time to expect to see changes in youth outcomes.

The spend-down strategy also led the Foundation to invest in national youth development organizations that it believed were stable enough to absorb significant investments and had the potential to sustain the work past the Foundation's funding. Lastly, the type of investments made were intended to build youth organizations' capacity such as to use data to improve trainings and programming or to improve national infrastructure including investments in technology and learning management systems. Capacity building was seen as a sustainable way to support organizations without pushing growth and scaling.

Focusing on improving adult practice is so crucial to offering high quality programs for youth and to developing a strong and supported workforce. Giving philanthropic support directly to youth serving organizations might temporarily serve more kids and improve access, but investing in youth workers is key to improving youth outcomes, and professionalizing what it is that they do will impact more than just youth today.

Founder and Board Chair Stephen D. Bechtel, Jr. was inspired to create the Foundation's national character initiative because he believed that high-quality programming and quality relationships with adult mentors are key to developing the skills youth need to be leaders. He 


\section{Betting on the Workforce}

personally had this experience through participation in the Boy Scouts and wants all youth to have similar opportunities.

To create the national character initiative, the Foundation first worked with a consulting group to conduct a field scan to develop recommendations for how the Foundation could make a significant impact investing in the YD field in a short amount of time in a way that would also be sustainable.

To amplify reach, the Foundation selected national organizations that fell into one of three cohorts. The leadership cohort comprises large youth-serving organizations like the Boys \& Girls Clubs of America (BGCA) and YMCA of the USA, which could serve millions of youth and were ready to launch quality improvement initiatives. The Foundation also developed a sports and play cohort focused on developing the skills of coaches to support character development and SEL. Lastly, the Foundation invested in a third cohort of organizations that use nature as a classroom and encourage environmental stewardship.

After identifying the organizations for investment, the Foundation made introductory, 1-year grants to get to know the organizations and better understand their capacity to influence their networks of affiliates and make system-level changes. These grants then resulted in multi-year investments (ranging from 2 to 4 years) that will end in 2020.

Are there commonalities among your grantees in how they have approached your charge to support the YD workforce?

Both: First, most grantees' strategies involve identifying best practices for SEL, building character, and developing or improving trainings so that staff are equipped to deliver these practices in their programs. This strategy focuses on elevating instinctively good practices from the workforce and then working with researchers to validate them. This highlights existing practices that are relevant to the uniqueness of each organization and reinforces to the YD workforce just how important their role is in identifying and reaching youth outcomes and organizational mission.

Second, almost all grantees proposed to build capacity in measurement, learning, and evaluation. Grantees were interested in collecting data to improve practice and ending data collection that was not useful. One interesting shift we observed is that when organizations built 


\section{Betting on the Workforce}

their staffs' capacity to use data, it moved the purpose of data collection out of fund development (to count and prove) and into the hands of field staff and managers so they could make meaning of it and improve programs.

An example of elevating organizational best practices is our work with Outward Bound. The system of 11 Outward Bound schools in the United States is supported by a national office (OBUSA) that protects the brand and charter and provides a variety of services for the schools. We worked with OBUSA to create the Outward Bound Professional Learning Lab. The lab's goal is to capture the best practices of seasoned instructors and translate these practices into existing and new trainings. Historically, the wisdom has been passed down from instructor to instructor. The lab is ensuring these practices are now accessible to all instructors.

From the data capacity-building side, OBUSA partnered with the PEAR Institute at Harvard University to validate the practices coming from instructors. PEAR Institute is also helping OBUSA develop an observation tool and youth outcomes instrument to support continuous quality improvement (CQI). This researcher-practitioner partnership has been effective for OBUSA and the rest of our grantees that share this strategy.

Rebecca, you support the "big nationals." Can you share an example of how a national grantee innovated to build capacity among their workforce?

Rebecca: When we began working with BGCA, their strategic plan was to improve the Club experience for youth across the country. After scaling clubs to reach as many youth as possible, the organization shifted its focus to improving the quality of clubs. BGCA implemented systems for collecting youth and staff data on youth outcomes and the Club experience and used that data to define the "optimal Club experience." With that understanding, BGCA set aggressive goals to double the percentage of Club members getting the optimal Club experience. The Foundation partnered with BGCA on this strategy, supporting the organization to raise awareness among Club staff of the importance of a high-quality Club experience, building the skills of staff at all levels, and creating a culture of CQI.

The key to this work is in the culture building. It is not an overnight process. The national office held town hall meetings across the country to engage Clubs and staff, released research briefs about the importance of the Club experience, revised and modernized its trainings and tools, and made its trainings and tools readily accessible to staff across the country. A great example 


\section{Betting on the Workforce}

of a revised tool is BGCA's Program Basics guidebook, last updated in the 1990s, now revised to align with BGCA's staff competencies and CQI strategy. Another example is the Youth Development Toolbox, which was created as an open-source app available to anyone working with youth. BGCA curates the content to ensure it is research-based and easily accessible. This has become a popular tool for frontline staff to help with lesson planning and on-the-spot activities.

BGCA is working toward its goal of increasing the percentage of Club members who receive the optimal Club experience and engaging in CQI. BGCA knows Clubs that participate in trainings, send multiple levels of staff to trainings, and complete the post-work, action planning, and youth surveys see the highest rate of improved Club experience. This shows BGCA is on the right track to improving youth outcomes by focusing on adult practice.

Alex, your grantees are the sports, nature, and play organizations. How have these YD organizations approached capacity building, and what can we learn from them?

Alex: Although most of the sports, nature, and play organizations we work with are approaching capacity building similarly to BGCA, there are a few unique approaches within the nature-based organizations. NatureBridge, Outward Bound, and the Student Conservation Association (SCA) share many of the programmatic elements of most YD organizations but are also responsible for keeping youth safe in the outdoors and creating a positive experience in nature. These latter elements often attract a workforce with stronger knowledge and skills related to the outdoors as opposed to YD.

SCA is known by many for its crews of young people building trails and protecting habitat in national parks around the country and less known as a YD model building strong leaders. SCA recently worked with the Search Institute to identify programmatic levers that lead to YD outcomes. They also enhanced trainings and professional development opportunities for their workforce to ensure that these outcomes are achieved. Like BGCA, SCA also has worked on building an internal workforce culture and external brand that champions SEL, leadership, and participants' conservation outcomes. Common language and ongoing communications are driving this culture change, resulting in a workforce that now identifies as both conservation leaders and YD leaders. 


\section{Betting on the Workforce}

SCA, NatureBridge, and Outward Bound presented at a handful of YD and after-school conferences this year, including BOOST and Ready by 21 . This is a big shift in the way these organizations identify as YD programs and surely will inspire YD organizations, researchers, and field leaders at these conferences. I hope this is the beginning of a cross-pollination of ideas between YD organizations and nature-based organizations, much like what I experienced at Golden Gate National Recreation Area.

You both touched upon the importance of culture change. What other lessons have you learned from your grantees that might be helpful to everyday YD organizations and programs?

\section{Both:}

- Changing culture-around YD workforce identity, CQI, data utilization, or partnershipstakes time, and so does managing that change. Be patient and keep at it-it will pay off in the long run.

- Elevate youth workers' self-awareness so that they understand how they relate to the youth they serve and how their work relates to the larger YD field. This was a key readiness step for sports, nature, and play organizations that did not see themselves in YD.

- Build awareness among staff on the importance of high-quality programming and how a CQI process can support adult practice to improve youth outcomes.

- Accept that CQI is an ongoing process, and that there is no silver bullet to improving quality. Organizations must adapt their strategies as they learn from their data and lessons from the field. Organizations also must commit to CQI for the long term to see demonstrable progress.

- Create a culture of using data as part of the CQI process to support and develop adult practice. Identify the data that will be most useful for building capacity of the YD workforce and focus your efforts there.

- Develop partnerships to expand access to trainings or professional development, identify common practices and guidelines, and/or develop measurement tools as needed. Or, better yet, look at commonly available resources and tools rather than starting from scratch.

AIR: Alex and Rebecca's lessons learned underscore several core components of a CQI process, such as a shared definition of quality and engaged stakeholders (Yohalem, Devaney, Smith, \& 


\section{Betting on the Workforce}

Wilson-Ahlstrom, 2012), and remind us that it requires "time, patience, persistence, and flexibility" (Spielberger, Axelrod Dasgupta, Cerven, Spain, Kohm, \& Mader, 2016, p. viii).

The Foundation used the model of communities of practice (CoPs) to convene grantees. We wanted to provide some background on CoPs before we move into Alex and Rebecca's responses.

Research suggests the value of CoPs for a variety of adult and youth outcomes (Fontaine, \& Millen, 2004). For example, Vescio, Ross, \& Adams (2008) found that well-developed CoPs have positive impacts on adult practice and youth outcomes. Studies have found that participants in CoPs demonstrated increased collaboration among participants, which meant that people had more colleagues to reach out to for support or guidance (Louis \& Marks, 1998), openness to having others observe their teaching/instruction and provide feedback (Phillips, 2003), and a culture of learning where people engaged in continuous quality improvement (Berry, Johnson, \& Montgomery, 2005). We asked,

\section{Why did you take a CoP approach and what have you learned from it?}

Both: We had experience with CoPs based on earlier experiences in our careers, so we know peer learning groups can support capacity building. We also saw the energy and interest in learning from one another when we first brought together grantees in 2016, so we formed CoPs based on staff roles to help grantees connect across organizations on issues they face on a daily basis. We support peer groups for the national CEOs as well as the organizational leads focused on strategy (COO/VPs), program quality, evaluation, and policy. The first few times they convened, facilitation focused on trust building and peer learning. As the group members jelled, they became more vocal about what they needed and wanted. The CEOs began trusting one another and asked to convene twice a year, which led to collaborative communications work to promote the importance of character development through each of their organizations' lenses, which will roll out in 2020.

We've seen the most energy from the evaluation peer group. Evaluation staff in YD organizations often are alone in that role and are not often brought together in peer-learning communities. Our evaluation CoP members are eager to spend time together and have chosen to host monthly calls between our larger in-person meetings. They also began presenting together and have developed cross-organizational partnerships through these gatherings. 
Another area of learning that has surfaced in the CoPs is exploring equity together-what does it look like in practice? How do inequitable systems and culture show up in the field and within our organizations? The Foundation convened two recent grantee meetings dedicated to diving into learning about equity and is continuing to work with and support the grantees on each of their diversity, equity, and inclusion strategies. None of us are experts in this work, but we can learn together.

All in all, we've seen at least a dozen meaningful organic cross-organizational partnerships come out of the CoPs and a genuine interest in collaborating-and this is among organizations that traditionally might see each other as competitors. Considering that the YD workforce is quite mobile and will move from one organization to another, it's important that these organizations continue to learn from one another, share training and professional development resources to support the YD workforce, and leverage each other's strengths to provide the highest quality programs for youth.

\section{What kind of impact do you hope the Foundation's investments have on YD and the workforce?}

Both: We believe that organizations have exemplar staff that are already implementing highquality YD practices. Our work focused on elevating those practices by validating them with research and working with professionals to develop trainings that equip more youth workers to understand and be prepared to implement those proven practices.

Rather than invest in new program development, the Foundation invested in building the internal capacity of these organizations to collect and learn from data and implement continuous improvement processes. With this group of national organizations really focused on improving program quality and providing the training and ongoing supports that the workforce needs to be successful with young people, we hope their work will have a larger impact on the YD field to continue investing in the workforce and sharing what works with one another. We're seeing many of these organizations talking to each other about sharing tools, trainings, and exploring common measures and certifications.

We have observed these national youth-serving organizations to:

- Look inward and use evaluation and data to inform better practices. 


\section{Betting on the Workforce}

- Make major improvements to trainings and training delivery systems (e.g., online, blended, learning management systems).

- Build awareness across their networks of affiliates of the importance of continuous quality improvement.

- Take a critical look at their internal policies and organizational culture to better support diversity, equity, and inclusion among staff (and ultimately the youth they serve), and determine what it will take to truly strive for equity.

- Increase energy toward partnering, collaborating, and learning from one another, whereas previously, they may have viewed one another as competitors.

The CEOs of the national organizations are working together on shared messaging, and that spirit of partnership trickles down through all levels of the organizations. Over this next year, we will support the grantees to share their work with the broader YD and education fields (and we will do the same with other funders) as what they're learning will be useful to the entire YD and education workforce.

Finally, we know the Foundation is sunsetting in 2020. What are your hopes for the YD field as we continue on in the spirit of your inspirational commitment? (Note: The interviewers added key resources or references for readers next to the lessons learned that are independent of the Foundation.)

Both:

- The field is talking a lot about whole child education and the importance of supporting youth in the in-school and out-of-school learning ecosystems (Whole Child Education, 2015; Darling-Hammond \& Cook-Harvey, 2018). Our hope is that after-school and OST organizations are key partners for $\mathrm{K}-12$ in reaching YD outcomes that they have mastered for decades, and that more $\mathrm{K}-12$ education funders see the value of investing in OST to support the whole child.

- The Science of Learning and Development tells us that relationships are key to learning (Science of Learning and Development Alliance, 2019). Most youth workers start with relationships (rather than academics) because this is what keeps youth engaged and helps them learn. The practice of building relationships is both instinctual and research based in YD and is a wonderful asset that the YD workforce can share more broadly with the education field. Learning happens everywhere, and we all need to work together to support young people's development and success. 


\section{Betting on the Workforce}

- We hope that the recommendations from the Aspen Institute's National Commission on Social, Emotional, and Academic Development will continue to elevate the important role that YD organizations play in supporting young people's success-they are critical partners to schools, families, and communities.

- We also hope the YD organizations we are working with continue their collaborative relationships-formally or informally. The CEOs already are talking about continuing to meet on their own after the sunset. The evaluation CoP is testing how to sustain its work together by self-facilitating monthly calls.

- Finally, we've never had a lot of data on who constitutes the YD workforce: What are their educational backgrounds, demographics, level of experience, career hopes, and so forth? It would be helpful to have a better understanding of who is in the workforce so that we can better support the workforce. Anecdotally and from some small studies, we know that frontline staff in these organizations often reflect the racial, ethnic, and socioeconomic diversity of the youth served. This is a huge asset of the YD workforce and a contrast to the teaching workforce that tends to struggle with diversifying to be more reflective of the student population. Despite the diversity of the YD workforce, however, there is much work to do to achieve that same level of diversity at the leadership levels in the field. It is critical that the YD (and education) field find ways to not only retain staff of color but to intentionally create pathways and support systems for staff of color to advance within the field. Some of our grantees are exploring strategies for creating more pathways to leadership within their organizations and are partnering with the CaISAC to learn from and implement elements of its Leadership Development Institute for Emerging Leaders of Color. More comprehensive data on the workforce could also be helpful when communicating to policymakers and funders the impact that YD has on youth, families, and communities.

\section{Closing}

Adults play a crucial role in young people's learning and development, including providing opportunities for SEL and character development (Bocarro and Witt, 2018; National Academies of Sciences, Engineering, and Medicine, 2019). Adult professionals establish and maintain highquality learning environments, and support youth through warm, stable, and responsive relationships (Durlak et al., 2011; Vandell et al., 2015). Rebecca and Alex are not dissimilar from many of us in the YD field; they value the YD workforce because they were members of it and see the impact it can have on young people. As members of the YD workforce, both Alex 
and Rebecca led or participated in sophisticated adult learning strategies, peer learning, and career pipeline development systems. They brought these experiences to their work at the Foundation. Their work with the Foundation connects research and practice by underscoring the importance of adult practice, CQI, meaningful data collection and use, and partnerships in achieving positive youth outcomes. We hope the strategies and lessons they shared in this interview inspire you to bet on the workforce, too.

\section{References}

American Institutes for Research. (2019). The Science of learning and development in afterschool systems and settings. https://www.air.org/resource/science-learning-and-developmentafterschool-systems-and-settings

Aspen Institute National Commission on Social, Emotional, and Academic Development. (2019). From a nation at risk to a nation at hope. http://nationathope.org/

Berry, B., Johnson, D., \& Montgomery, D. (2005). The power of teacher leadership. Educational Leadership, 62(5), 56-60. ASCD. http://www.ascd.org/publications/educationalleadership/feb05/vol62/num05/The-Power-of-Teacher-Leadership.aspx

Bocarro, J. N., \& Witt, P. A. (2018). The power of people: The importance of relationship-based programming. In P. A. Witt \& L. L. Caldwell (Eds.), Youth development: Principles and practices in out-of-school time settings ( $2^{\text {nd }}$ ed.) (pp. 389-404). Urbana, IL: Sagamore-Venture.

Borden, L. M., \& Perkins, D. F. (2006). Community youth development professionals: Providing the necessary supports in the United States. Child \& Youth Care Forum, 35(2), pp. 101-158.

Caldwell, L. I., \& Witt, P. A. (2018). Ten principles of youth development. In P. A. Witt \& L. L. Caldwell (Eds.), Youth development: Principles and practices in out-of-school time settings ( $2^{\text {nd }}$ ed.) (pp. 1-26). Urbana, IL: Sagamore-Venture.

Darling-Hammond, L., \& Cook-Harvey, C. (2018). Educating the whole child: Improving school climate to support student success. https://learningpolicyinstitute.org/product/educating-whole-child-report

Darling-Hammond, L., Flook, L., Cook-Harvey, C., Barron, B., \& Osher, D. 2019. Implications for educational practice of the science of learning and development. Applied Developmental Science, https://doi.org/10.1080/10888691.2018.1537791

Durlak, J. A., Weissberg, R. P., Dymnicki, A. B., Taylor, R. D., \& Schellinger, K. B. (2011). The impact of enhancing students' social and emotional learning: A meta-analysis of school-based universal interventions. Child Development, 82(1), 405-432. https://casel.org/wpcontent/uploads/2016/06/meta-analysis-child-development-1.pdf.

Fontaine, M. \& Millen, D. (2004). Chapter I Understanding the Benefits and Impact of Communities of Practice. 


\section{Betting on the Workforce}

Louis, K. S., \& Marks, H. M. (1998). Does professional learning community affect the classroom? Teachers' work and student experiences in restructuring schools. American Journal of Education, 106(4), 532-575. https://doi.org/10.1086/444197

Moroney, D. A., \& Devaney, E. (2017). Ready to implement? How the out-of-school time workforce can support character development through social and emotional learning: A Review of the literature and future directions. Journal of Character Education, 13(1), 67-89.

Moroney, D., Newman, J., \& Osher, D. (2018). Out-of-school-time programs. In D. Osher, D. Moroney, \& S. Williamson (Eds.), Creating safe, equitable, engaging schools: $A$ comprehensive, evidencebased approach to supporting students (pp. 121-134). Cambridge, MA: Harvard Education Press.

National Academies of Sciences, Engineering, and Medicine. (2019). Shaping summertime experiences: Opportunities to promote healthy development and well-being for children and youth. Washington, DC: The National Academies Press. https://doi.org/10.17226/25546

Phillips, J. (2003). Powerful learning: Creating learning communities in urban school reform. Journal of Curriculum and Supervision, 18(3), 240-258.

Science of Learning and Development Alliance. (2019). How the science of learning and development can transform education: Initial findings. https://www.soldalliance.org/resources

Spielberger, J., Axelrod, J., Dasgupta, D., Cerven, C., Spain, A., Kohm, A., \& Mader, N. (2016). Connecting the dots: Data use in afterschool systems. Chicago, IL: Chapin Hall at the University of Chicago.

Vandell, D. L., Larson, R. W., Mahoney, J. L., \& Watts, T. W. (2015). Children's organized activities. In M. H. Bornstein, T. Leventhal, \& R. M. Lerner (Eds.), Handbook of child psychology and developmental science: Ecological settings and processes in developmental systems (pp. 305344). Hoboken, NJ: Wiley.

Vescio, V., Ross, D., \& Adams, A. (2008). A review of research on the impact of professional learning communities on teaching practices and student learning. Teaching and Teacher Education, 24(1), pp. 80-91. https://doi.org/10.1016/j.tate.2007.01.004

Whole Child Education. (2015). The whole child approach to education.

\section{http://www.wholechildeducation.org/about}

Yohalem, N., Devaney, E., Smith, C., \& Wilson-Ahlstrom, A. (2012). Building citywide systems for quality: A guide for afterschool leaders. Washington, DC: The Forum for Youth Investment. 